\title{
MMP1 Is a Prognostic-Related Biomarker and Correlated with Immune Infiltration in Breast Cancer
}

\author{
Xiangdong Wei*, Bin Zhang, Baojun Pan \\ Changle People's Hospital, Weifang, China \\ Email: *13963608206@163.com
}

How to cite this paper: Wei, X.D., Zhang, B. and Pan, B.J. (2022) MMP1 Is a Prognostic-Related Biomarker and Correlated with Immune Infiltration in Breast Cancer. Health, 14, 219-235.

https://doi.org/10.4236/health.2022.142017

Received: December 3, 2021

Accepted: February 22, 2022

Published: February 25, 2022

Copyright $\odot 2022$ by author(s) and Scientific Research Publishing Inc. This work is licensed under the Creative Commons Attribution International License (CC BY 4.0).

http://creativecommons.org/licenses/by/4.0/ (c) (i) Open Access

\section{Abstract}

Background: Matrix metalloproteinases 1 (MMP1) plays a role in cancer development and metastasis and high expression of MMP1 has been confirmed in various types of cancers. However, the correlation between MMP1 and prognosis and tumor-infiltration lymphocytes in breast cancer remains uncertain. In this present study, we analyzed MMP1 expression and correlation with prognosis of cancer patients in databases such as Oncomine, PrognoScan, and Kaplan-Meier plotter. In addition, we investigated the correlation of MMP1 with tumor-infiltrating immune cells in the different tumor microenvironments via Tumor Immune Estimation Resource (TIMER). Methods: MMP1 expression was analyzed via the Oncomine database and Tumor Immune Estimation Resource (TIMER) site. The prognosis of MMP1 on cancers was analyzed using Kaplan-Meier plotter, the PrognoScan database and Gene Expression Profiling Interactive Analysis (GEPIA). The correlations between MMP1 and cancer immune infiltration were investigated by TIMER. In addition, correlations betweenMMP1 expression and gene marker sets of immune infiltration were analyzed by TIMER and GEPIA. Results: MMP1 is highly expressed in most cancers and correlated to poor prognosis. MMP1 expression is significantly linked with a poorer prognosis in breast cancer (OS HR $1.78,95 \% \mathrm{CI}=1.59-1.98, \mathrm{P}<0.0001$; RFS HR $1.82,95 \% \mathrm{CI}=1.19-2.80, \mathrm{P}=$ 0.0053 ) and breast cancer (OS HR 2.63, $\mathrm{P}=0.02$ ). In addition, the expression of MMP1 was significantly associated with levels of $\mathrm{CD}^{+} \mathrm{T}$ cell $(\mathrm{R}=0.464, \mathrm{P}$ $=2.97 \mathrm{e}-54), \mathrm{CD}^{+} \mathrm{T}$ cell $(\mathrm{R}=-0.134, \mathrm{P}=2.17 \mathrm{e}-05)$, macrophage $(\mathrm{R}=0.41, \mathrm{P}$ $=1.11 \mathrm{e}-08)$, dendritic cell $(\mathrm{R}=0.221, \mathrm{P}=2.92 \mathrm{e}-03)$ and $\mathrm{NK}$ cell $(\mathrm{R}=0.213, \mathrm{P}$ $=4.15 \mathrm{e}-03)$. Besides, MMP1 expression is significantly associated with the marker genes of immune cells $(\mathrm{P}<0.001)$. Conclusions: Our study indicates that MMP1 is correlated with prognosis and immune infiltrating levels of $\mathrm{CD}^{+} \mathrm{T}$ cell, $\mathrm{CD}^{+} \mathrm{T}$ cell, macrophage, dendritic cell and NK cells in breast 
cancer. Besides, MMP1 expression potentially contributes to regulation of $\mathrm{T}$ cell, B cell, tumor-associated macrophages (TAMs), DCs, T cell exhaustion and Tregs in colon and gastric cancer. The results indicate that MMP1 can be used as a prognostic biomarker for determining prognosis and immune infiltration in breast cancer.

\section{Keywords}

MMP1, Breast Cancer, Prognosis, Tumor-Infiltration

\section{Introduction}

Esophagus cancer remains an important cancer worldwide and is responsible for over 572,034 new cases in 2018 and an estimated 508,585 deaths, which is the ninth most frequently diagnosed cancer [1]. Since most patients diagnosed with early esophageal cancer lack any symptoms before the onset of dysphagia and weight loss that can signal an advanced-stage tumor, esophagus cancer has a particularly poor prognosis [2] [3] [4]. Therefore, it's urgently needed to investigate a new target for esophagus diagnosis and treatment.

Breast cancer is the most prevalent cancer in women (22\% of the new cases) and is also the most common cause of death from cancer in the majority of countries (154 of 185) [1]. There will be about 2.1 million newly diagnosed female breast cancer cases in 2018, accounting for almost 1 in 4 cancer cases among women. In addition, the incidence rates of breast cancer are increasing in most countries over the last decades. Even though the treatments in breast cancer have been improved which inducing longer 5-year survival, the breast cancer remains the major cause of death [5] [6] [7]. Therefore, the primary prevention of breast cancer remains one of the most effective strategies to reduce breast cancer burden [8].

Matrix metalloproteinases (MMPs) are a group of proteolytic enzymes that can degrade extracellular matrix components, which can affect various physiological and pathological processes such as cell apoptosis, angiogenesis, tissue repair, immune response and tumor progression and invasiveness [9] [10] [11]. It has been identified that specific MMP can promote or inhibit tumorigenesis and/or metastasis, depending on the tumor type, cellular source of expression and disease stage. MMP-1, also termed collagenase-1 or interstitial collagenase, which can degrade collagen and gelatin. MMP-1 expression is augmented by inflammatory cytokines such as tumor necrosis factor- $\alpha$ (TNF- $\alpha$ ) and interleukin-1 (IL-1). MMP-1 may play a role in cancer development and metastasis. High expression of MMP1 has been confirmed in various types of cancers, and is associated with unfavorable clinical outcome in malignancies such as esophageal cancer [12], hepatocellular carcinoma [13], gastric cancer [14], gallbladder carcinoma [15], thyroid carcinoma [16], pancreatic carcinoma [17] and colorectal cancers [18]. 
In this present study, we analyzed MMP1 expression and correlation with prognosis of cancer patients in databases such as Oncomine, PrognoScan, and Kaplan-Meier plotter. In addition, we investigated the correlation of MMP1 with tumor-infiltrating immune cells in the different tumor microenvironments via Tumor Immune Estimation Resource (TIMER). Our results showed the potential mechanism of MMP1 influence immune cell infiltration and provide new insight into MMP1 as a targeting site for treating breast cancer.

\section{Material and Methods}

\subsection{Oncomine Database Analysis}

The expression level of the MMP1 gene in various types of cancers was identified in the Oncomine database (https://www.oncomine.org/resource/login.html). Oncomine, a cancer microarray database, aims to stimulate new discovery from genome-wide expression analyses and compare the transcriptome data in most major types of cancer with respective normal tissues [19] [20]. To date, ONCOMINE contains 715 gene expression datasets comprising nearly 86,733 samples. In this study, we selected 1.5 -fold change, p-value $=0.05$, and top $10 \%$ gene rank as threshold.

\subsection{PrognoScan Database Analysis}

The correlation between MMP1 expression and survival in various types of cancers was analyzed by the PrognoScan database (http://www.abren.net/PrognoScan/). PrognoScan database contains a large collection of publicly available cancer microarray datasets with clinical annotation, which assess the biological relationship between gene expression and prognosis [21] [22]. PrognoScan employs the minimum P-value approach for grouping patients for survival analysis, in this study, the threshold was adjusted to a Cox $\mathrm{P}$-value $<0.05$.

\subsection{Kaplan-Meier Plotter Database Analysis}

The correlation between MMP1 expression and survival in breast, ovarian, lung and gastric cancers was analyzed by Kaplan-Meier plotter (http://kmplot.com/analysis/). Kaplan-Meier plotter is an online database including gene expression data and clinical data, which contains breast cancer [23], lung cancer [24], ovarian cancer [25] and gastric cancer [26]. In this study, we analyzed the overall survival (OS) and RFS of breast, ovarian, lung and gastric cancers patients by using a Kaplan-Meier survival plot. The hazard ratio (HR) with 95\% confidence intervals and log-rank P-value were also computed.

\subsection{TIMER Database Analysis}

The MMP1 expression in different types of cancer and the correlation of MMP1 expression with immune infiltrates, including $\mathrm{B}$ cells, $\mathrm{CD} 8^{+} \mathrm{T}$ cells, $\mathrm{CD} 8^{+} \mathrm{T}$ cells, Treg, neutrophils, macrophages, and dendritic cells were analyzed in TIMER 
database via gene modules. In addition, the correlations between MMP1 expression and gene markers of tumor-infiltrating immune cells were analyzed via correlation modules. TIMER2.0 (http://timer.cistrome.org/) web server provides comprehensive analysis and visualization functions of tumor infiltration immune cells [27]. TIMER provides more robust estimation of immune infiltration levels for The Cancer Genome Atlas (TCGA) or user-provided tumor profiles using six state-of-the-art algorithms with four modules.

In this study, we analyzed the gene markers of tumor-associated macrophages (TAM), M1 macrophages, M2 macrophages, neutrophils, natural killer (NK) cells, $\mathrm{CD}^{+} \mathrm{T}$ cells, $\mathrm{T}$ cells, B cells, monocytes, dendritic cells (DCs), T-helper 1 (Th1) cells, T-helper 2 (Th2) cells, follicular helper T (Tfh) cells, T-helper 17 (Th17) cells, Tregs, and exhausted $\mathrm{T}$ cells. In addition, the correlation module generated the expression scatter plots between a pair of user-defined genes in a given cancer type, together with the Spearman's correlation and the estimated statistical significance. MMP1 was used for the $\mathrm{x}$-axis with gene symbols, and related marker genes are represented on the $y$-axis as gene symbols. The gene expression level was displayed with $\log 2$ RSEM.

\subsection{Gene Correlation Analysis in GEPIA}

The survival curves, including OS and DFS, based on gene expression with the log-rank test and the Mantel-Cox test in 33 different types of cancer were generated in the online database Gene Expression Profiling Interactive Analysis (GEPIA) (http://gepia.cancer-pku.cn/index.html). In addition, GEPIA was used to further confirm the significantly correlated genes in TIMER. GEPIA is an interactive web application for gene expression analysis based on 9736 tumors and 8587 normal samples from the TCGA and the GTEx databases, which provides key interactive and customizable functions including differential expression analysis, profiling plotting, correlation analysis, patient survival analysis, similar gene detection and dimensionality reduction analysis [28]. In this study, the Spearman method was used to determine the correlation coefficient. MMP1 was used for the $\mathrm{x}$-axis, and other genes of interest are represented on the $\mathrm{y}$-axis. The tumor and normal tissue datasets were used for analysis.

\subsection{Statistical Analysis}

Survival curves were generated by the PrognoScan and Kaplan-Meier plots. The results generated in Oncomine are displayed with P-values, fold changes, and ranks. The results of Kaplan-Meier plots, PrognoScan, and GEPIA are displayed with HR and P or Cox P-values from a log-rank test. The correlation of gene expression was evaluated by Spearman's correlation and statistical significance, and the strength of the correlation was determined using the following guide for the absolute value: 0.00 - 0.19 "very weak", 0.20 - 0.39 "weak", 0.40 - 0.59 "moderate", 0.60 - 0.79 "strong", $0.80-1.0$ "very strong". P-values $<0.05$ was considered statistically significant. 


\section{Results}

\subsection{Assessment the mRNA Expression Level of MMP1 in Different Human Cancer and Normal Tissue}

To determine differences of MMP1 expression in tumor and normal tissues, we assessed the LAYN mRNA levels in different tumors and normal tissues of multiple cancer types using the Oncomine database. The results showed that the MMP1 expression was higher in bladder, breast, cervical, colorectal, esophageal, gastric, head and neck, lung cancers compared to the normal tissue (Figure 1A). Besides, relative to the normal tissues, MMP1 expression was lower in brain and CNS cancer.

We further examined MMP1 expression using the RNA-seq data of multiple malignancies in TCGA and TIMER database. The results showed that MMP1 expression was significantly higher in bladder urothelial carcinoma (BLCA), breast invasive carcinoma (BRCA), CESC, cholangiocarcinoma (CHOL), colon adenocarcinoma (COAD), esophagus cancer (ESCA), glioblastoma multiforme (GBM), head and neck cancer (HNSC), kidney renal clear cell carcinoma (KIRC), liver hepatocellular carcinoma (LIHC), lung adenocarcinoma (LUAD), lung squamous cell carcinoma (LUSC), pancreatic adenocarcinoma (PAAD), rectum adenocarcinoma (READ), skin cutaneous melanoma (SKCM), stomach adenocarcinoma (STAD), thyroid carcinoma (THCA), uterine corpus endometrial carcinoma (UCEC). However, MMP1 expression was significantly lower in kidney chromophobe $(\mathrm{KICH})$, kidney renal papillary cell carcinoma (KIRP) compared to normal tissue (Figure 1B).

\subsection{The Correlation between LAYN Expression and Prognosis in Cancer Patients}

In order to determine the correlation between LAYN expression and prognosis in cancer patients, we examined the underlying mechanisms via using the PrognoScan database. The results showed that MMP1 expression impacts poor prognosis in 5 types of cancers, including breast, ovarian, head and neck, esophagus cancers (Figures 2A-N). In addition, we employed the Kaplan-Meier plotter database to assess how MMP1 expression relates to prognosis in a range of cancer types, revealing its elevation to be significantly linked with a poorer prognosis in breast cancer (OS HR 1.78, 95\% CI $=1.59-1.98, \mathrm{P}<{ }^{*} 1$; RFS HR $1.82,95 \% \mathrm{CI}=1.19-2.80, \mathrm{P}=1.97 \mathrm{e}-7 ; \mathrm{P}=0.0053$ ) (Figures $2 \mathrm{G}-\mathrm{H}$ ). However, the correlation between ovarian cancer (OS HR 1.78, 95\% CI $=1.59-1.98, \mathrm{P}<$ ${ }^{* *} 1$; RFS HR 1.82, 95\% CI $\left.=1.19-2.80, \mathrm{P}=1.97 \mathrm{e}-7 ; \mathrm{P}=0.0053\right)$ and gastric cancer (OS HR 1.78, 95\% CI $=1.59-1.98, \mathrm{P}<{ }^{* *} 1$; RFS HR 1.82, 95\% CI $=1.19$ $2.80, \mathrm{P}=1.97 \mathrm{e}-7 ; \mathrm{P}=0.0053$ ) were reduced. Notably, there was no significant relationship between the expression of MMP1 expression and the prognosis of lung cancer patients (Figures 2O-P). Therefore, it is conceivable that high LAYN expression is an independent risk factor and leads to a poor prognosis in breast patients. 


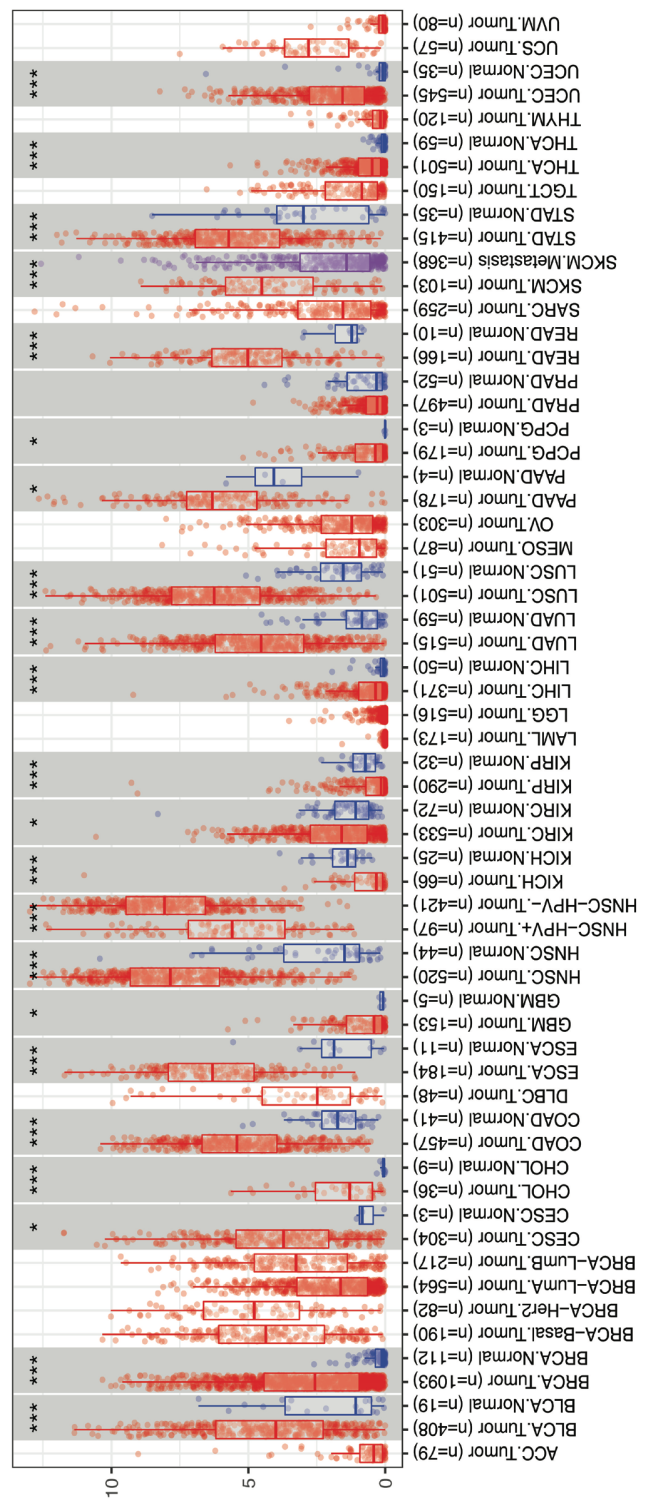

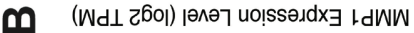
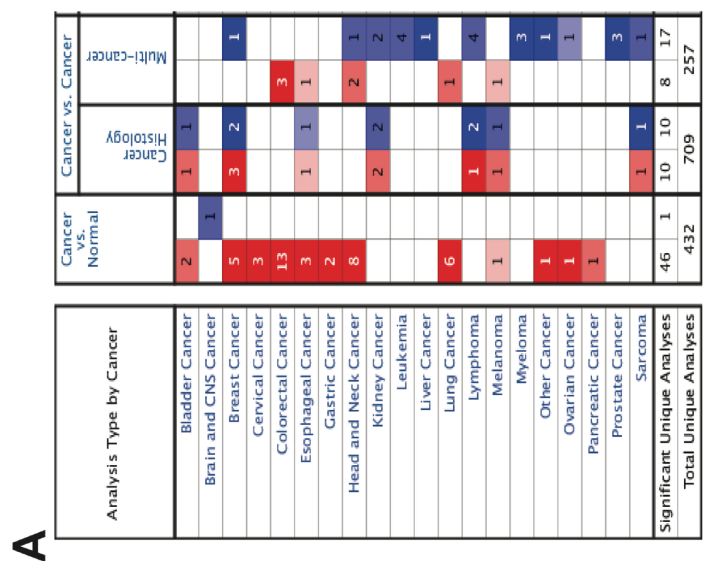

Figure 1. MMP1expression level in different human tumor and normal tissues. (A) The expression level of MMP1 in different types of tumor tissues and normal tissues in the Oncomine database. ( $\mathrm{P}$ value is 0.001 , fold change is 1.5 , and gene ranking of all. (B) MMP1 expression level in different types of tumor tissues and normal tissues in TIMER database $\left({ }^{\star} \mathrm{P}<0.05,{ }^{\star \star} \mathrm{P}<0.01,{ }^{\star *} \mathrm{P}<0.001\right)$. 
A Breast cancer GSE1456 DSS

$\mathrm{HR}=1.41 \operatorname{Cox} P=0.0003$

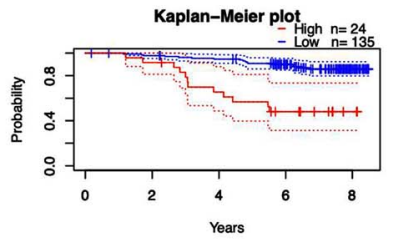

D

Esophagus cancer GSE11595 OS $\mathrm{HR}=2.63 \operatorname{Cox} P=0.02$

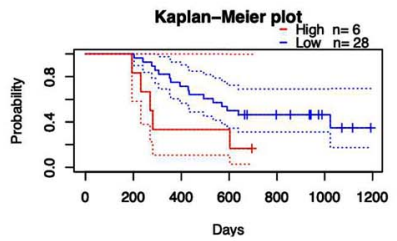

B Ovarian cancer GSE17260 PFS

$\mathrm{HR}=0.87 \operatorname{Cox} P=0.006$

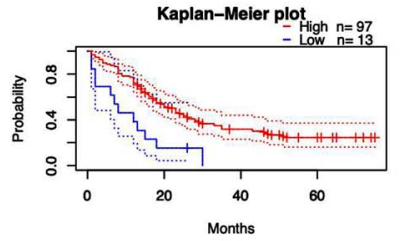

E

Colorectal cancer GSE14333 DFS $\mathrm{HR}=0.88 \operatorname{Cox} P=0.10$

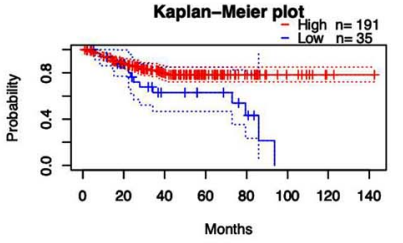

K
C Head and neck cancer GSE2837 RFS $\mathrm{HR}=1.32 \operatorname{Cox} P=0.01$

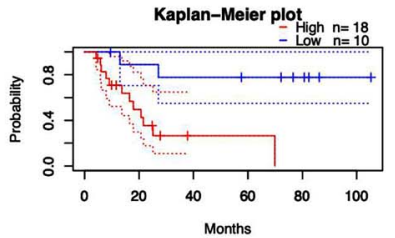

$\mathbf{F}$

Lung cancer GSE3141 OS $\mathrm{HR}=1.14 \operatorname{Cox} P=0.11$

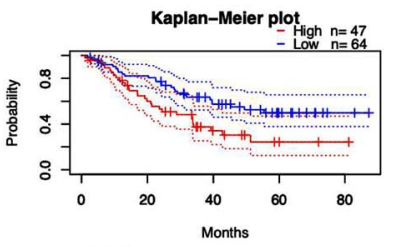

M
G

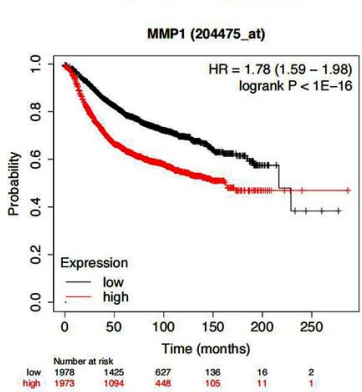

H

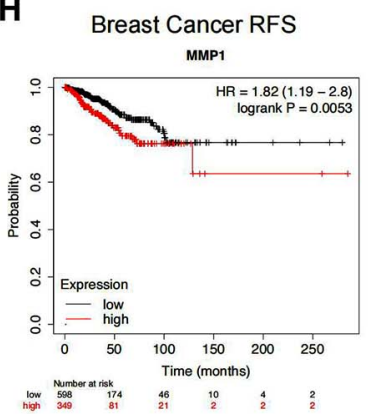

I

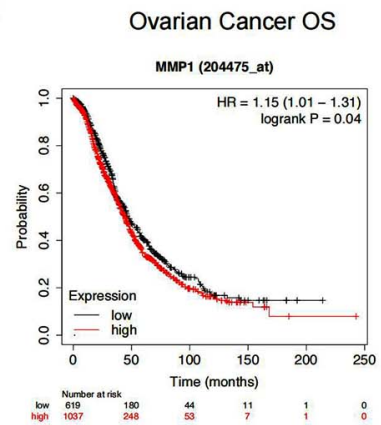

J

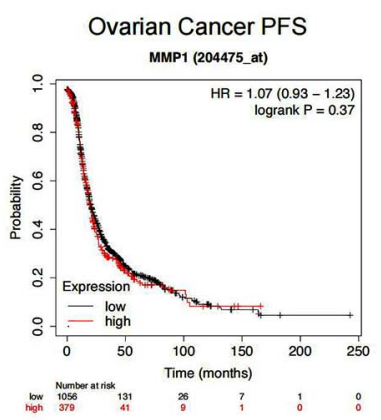

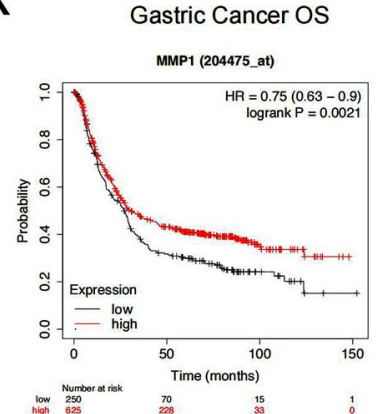

$\mathbf{L}$

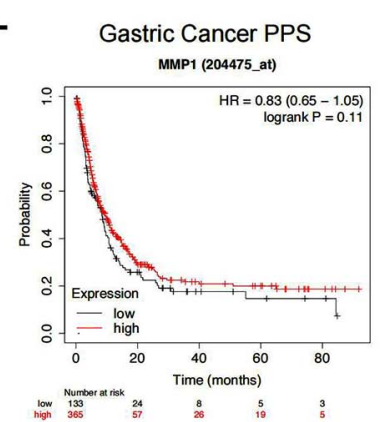

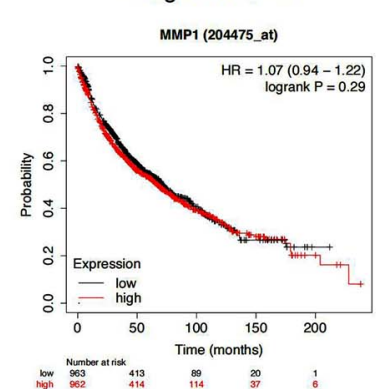

$\mathbf{N}$

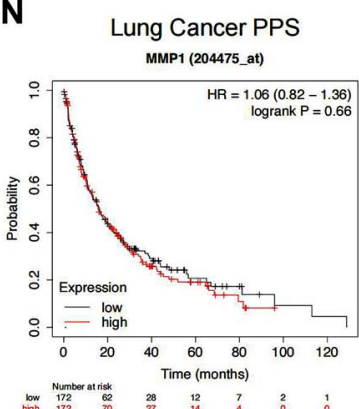

Figure 2. Correlation between MMP1 and prognosis of different in the PrognoScan (A-F) and Kaplan-Meier plotter databases (G-N). (A-F) Survival curves of DSS, PFS, RFS, OS, DFS and OS in breast, ovarian, head and neck, esophagus, colorectal and lung cancer. (G, H) OS and RFS survival curves of breast cancer $(n=3951, n=945)$. (I, J) OS and PFS survival curves of ovarian cancer $(\mathrm{n}=1656, \mathrm{n}=1059)$. (K, L) OS and PPS survival curves of gastric cancer $(\mathrm{n}=875, \mathrm{n}=498)$. (M, N) OS and PFS survival curves of lung cancer $(\mathrm{n}=847, \mathrm{n}=344)$. OS, overall survival; DFS, disease-free survival; RFS, relapse-free survival; DSS, disease-specific survival.

\subsection{High MMP1 Expression Relates to Prognosis in Breast Cancer Patients}

To explore the relevance and underlying mechanisms MMP1 expression in cancer, we examined the relationship between the MMPlexpression and clinical characteristics of breast cancer patients in the Kaplan-Meier plotter databases. Breast cancers are heterogenous, showing variable morphologic and biological 
features, including estrogen receptor (ER), progesterone receptor (PR), and human epidermal growth factor receptor 2 (HER2) expression of immunophenotype [29]. The results showed that overexpression of MMP1 was associated with worse OS and PFS in breast patients $(\mathrm{P}<0.05)$, and with ER positive, HER2 negative, intrinsic luminal A, lymph node statues and grade 2 (Table 1 ). In addition, the staging of breast cancers is based on tumor size, nodal status, and distant metastasis (TNM staging). Grading is a powerful prognostic factor and serves as an integral component in a number of clinical decision tools such as the Nottingham prognostic index and Adjuvant online [30]. These results indicate that MMP1 expression is associated with the prognosis of breast cancer.

\subsection{MMP1 Expression Correlated with Immune Infiltration Level in Breast and Esophagus Cancer}

Survival and lymph node metastasis are independently predicted by the frequency of lymphocytes infiltrating into the tumor in cancers. Furthermore, we examined the correlation between MMP1 expression and immune infiltration levels in different types of cancer by using TIMER database. The results showed the expression in ESCA tumor has a significant correlation with purity, B cell, $\mathrm{CD}^{+} \mathrm{T}$ cell, $\mathrm{CD}^{+} \mathrm{T}$ Cell, macrophage, neutrophil, dendritic cell and NK cell (Figure 3A). Unlike the ESCA, the MMP1 expression has no significant correlation with purity and B cell in BRCA, whereas in this same tumor type, the

Table 1. Correlation of MMP1 mRNA expression and clinical prognosis in breast cancer with different clinicopathological factors by Kaplan-Meier plotter.

\begin{tabular}{|c|c|c|c|c|c|c|c|}
\hline \multirow{2}{*}{\multicolumn{2}{|c|}{$\begin{array}{l}\text { Clinicopathological } \\
\text { characteristics }\end{array}$}} & \multicolumn{3}{|c|}{ Overall survival $(\mathrm{n}=1402)$} & \multicolumn{3}{|c|}{ Rrgerreion-free survival ( $\mathrm{n}=3951$ ) } \\
\hline & & $\mathrm{N}$ & Hazard ration & P-value & $\mathrm{N}$ & Hazard ration & $\mathrm{P}$-value \\
\hline \multirow{2}{*}{ ER } & positive & 2565 & $1.61(1.24-2.09)$ & $* *$ & 2565 & $1.61(1.37-1.90)$ & $* * *$ \\
\hline & negative & 1214 & $1.10(0.75-1.63)$ & 0.6200 & 1214 & $1.16(0.93-1.46)$ & 0.1900 \\
\hline \multirow{2}{*}{$\mathrm{PR}$} & postive & 954 & $1.34(0.35-5.09)$ & 0.6700 & 954 & $1.36(0.96-1.93)$ & 0.0811 \\
\hline & negative & 1028 & $1.01(0.40-2.55)$ & 0.9800 & 1028 & $1.06(0.79-1.42)$ & 0.6969 \\
\hline \multirow{2}{*}{ HER2 } & postive & 416 & $1.25(0.62-2.50)$ & 0.5300 & 416 & $0.94(0.61-1.45)$ & 0.7917 \\
\hline & negative & 1456 & $2.43(0.94-6.28)$ & 0.0580 & 1456 & $1.86(1.42-2.44)$ & $* * *$ \\
\hline \multirow{4}{*}{ Intrinsic subtype } & basal & 879 & $1.65(1.00-2.71)$ & 0.0480 & 879 & $1.20(0.94-1.55)$ & 0.1483 \\
\hline & luminal A & 2504 & $1.72(1.20-2.47)$ & 0.0028 & 2504 & $1.89(1.58-2.25)$ & $* * *$ \\
\hline & luminal B & 1425 & $1.30(0.90-1.89)$ & 0.1600 & 1425 & $1.28(1.06-1.55)$ & 0.0113 \\
\hline & HER2+ & 335 & $0.57(0.29-1.11)$ & 0.0940 & 335 & $0.76(0.52-1.12)$ & 0.1593 \\
\hline \multirow{2}{*}{$\begin{array}{l}\text { lymph node } \\
\text { status }\end{array}$} & postive & 1459 & $1.13(0.76-1.66)$ & 0.5500 & 1459 & $1.67(1.37-2.03)$ & $* * *$ \\
\hline & negative & 2259 & $2.00(1.36-2.92)$ & ** & 2259 & $1.63(1.37-1.93)$ & $* * *$ \\
\hline \multirow{3}{*}{ Grade } & 1 & 378 & $1.94(0.76-4.94)$ & 0.1600 & 378 & $1.50(0.89-2.52)$ & 0.1242 \\
\hline & 2 & 1077 & $1.75(1.13-2.70)$ & 0.0110 & 1077 & $1.60(1.26-2.05)$ & ** \\
\hline & 3 & 1099 & $1.10(0.80-1.53)$ & 0.5600 & 1090 & $1.13(0.91-1.40)$ & 0.2809 \\
\hline \multirow{2}{*}{ TP53 status } & mutated & 232 & $0.53(0.24-1.19)$ & 0.1200 & 232 & $0.84(0.59-1.51)$ & 0.7969 \\
\hline & wild type & 363 & $1.77(0.91-3.45)$ & 0.0870 & 363 & $1.40(0.91-2.13)$ & 0.1204 \\
\hline
\end{tabular}


expression of MMP1 was significantly associated with levels of $\mathrm{CD} 8^{+} \mathrm{T}$ cell $(\mathrm{R}=$ 0.464, $\mathrm{P}=2.97 \mathrm{e}-54), \mathrm{CD} 8^{+} \mathrm{T}$ cell $(\mathrm{R}=-0.134, \mathrm{P}=2.17 \mathrm{e}-05)$, Macrophage $(\mathrm{R}=$ $0.41, \mathrm{P}=1.11 \mathrm{e}-08)$, dendritic cell $(\mathrm{R}=0.221, \mathrm{P}=2.92 \mathrm{e}-03)$ and $\mathrm{NK}$ cell $(\mathrm{R}=$ $0.213, \mathrm{P}=4.15 \mathrm{e}-03$ ) (Figure $3 \mathrm{~B}$ ). In addition, the MMP1 expression is correlated to purity, B cell, $\mathrm{CD} 8^{+} \mathrm{T}$ cell, macrophage, neutrophil, and NK cell, except of $\mathrm{CD}^{+} \mathrm{T}$ Cell and dendritic cells in STAD (Figure 3C).

Further, the results showed that MMP1 expression level correlate with poorer prognosis and high.

immune infiltration in ESCA and BRCA. These results indicate that MMP1 plays a specific role in immune infiltration in breast and esophagus cancers, especially those of macrophage and $\mathrm{CD}^{+} \mathrm{T}$ cell.

\subsection{Analysis of the Correlation between MMP1 and Immune Marker Expression}

To determine the relationship between MMP1 and the diverse immune infiltrating cells, we examined the correlations between MMP1and immune marker sets of various immune cells of ESCA and BRCA using TIMER and GEPIA databases, with STAD as a control group. The $\mathrm{CD} 8^{+} \mathrm{T}$ cells, $\mathrm{T}$ cells, B cells, monocytes, TAMs, M1 and M2 macrophages, neutrophils, NK cells, DCs, Th1 cells, Th2 cells, Tfh cells, Th17 cells, Tregs and exhausted T cells, in ESCA and BRCA were analyzed (Table 2), the results were adjusted based on tumor purity. The results revealed the MMP1 expression level was significantly correlated with most immune marker sets of various immune cells and different $\mathrm{T}$ cells in BRCA. In contrast, the MMP1 expression level was significantly correlated with only 12 gene markers in ESCA and 13 gene markers in STAD.

Notably, the MMP1 expression level was significantly correlated with $\mathrm{CD} 8^{+} \mathrm{T}$ cell, T cells, B cells, TAMs, M1 macrophages, neutrophiles, dendritic cells and Treg in BRCA and ESCA. Specifically, CD8B of CD8 ${ }^{+} \mathrm{T}$ cell, CD3D, CD3E, CD2 of T cell, CD19, CD79A of B cell, chemokine (C-C motif) ligand (CCL)-2, IL10 of TAMs, NOS2, PTGS2 of M1 macrophages, CCR7 of neutrophils, HLA-DQB1 of dendritic cell, TGF $\beta$ of Treg are significantly correlated with MMP1 expression in BRCA and ESCA $(\mathrm{P}<0.05)$ (Figure 4). Further, the correlation between MMP1 and the above gene markers were analyzed in GEPIA database, including BRCA, ESCA and STAD. The results are similar to these in TIMER (Table 3). These results indicate that MMP1 may regulate the T cell, Treg, B cell and macrophage polarization and activate $\mathrm{T}$ cell, $\mathrm{B}$ cell and Treg in BRCA and ESCA.

In addition, MMP1 expression is linked with the DC markers HLA-DQB1, HLA-DRA, HLA-DPA, which suggested that MMP1 is associated with DC infiltration. DCs are able to increase levels of tumor metastasis via enhancing Treg responses and suppressing $\mathrm{CD} 8^{+} \mathrm{T}$ cell cytotoxicity, which consist with the high level of Treg markers FOXP3, CCR8, STAT5B, TGF $\beta$. Besides, recruiting Treg cells into tumor is a potent immunosuppressive mechanism in a variety of cancer types. However, further researchers are needed to confirm the role of MMP1 in regulating DC, Treg and tumor progression. 


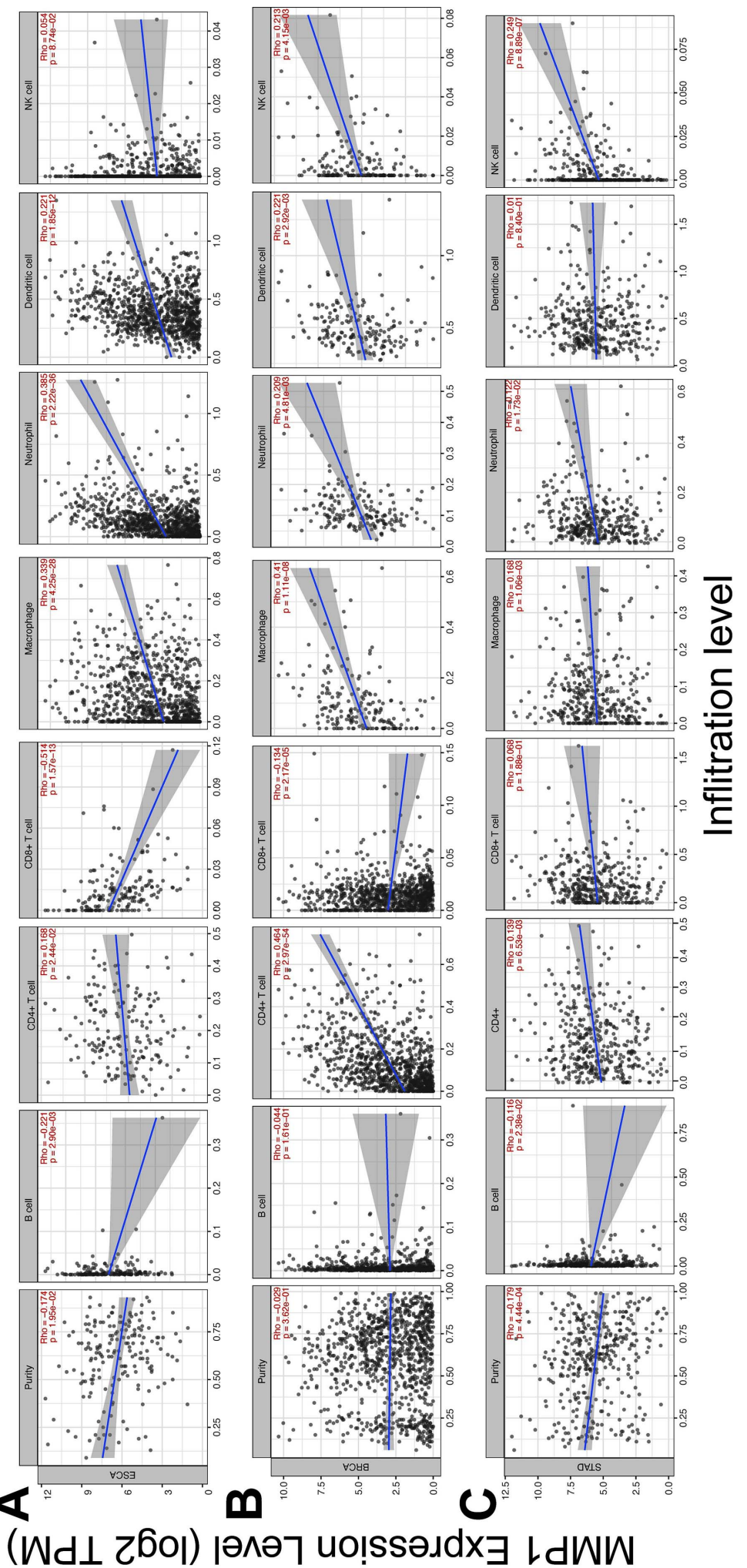

Figure 3. Correlation between MMP1 expression with immune infiltration level in ESCA, BRCA and STAD. (A, B) MMP1 expression is significantly negatively related to tumor purity, $\mathrm{B}$ cell and $\mathrm{CD} 8^{+} \mathrm{T}$ cells and has significantly positive correlations with infiltrating levels of $\mathrm{CD}^{+} \mathrm{T}$ cells, macrophages, neutrophils, and dendritic cells in ESCA and BRCA. (C) MMP1 expression is significantly negatively related to tumor purity and B Cell, and has significant positive correlations with infiltrating levels of CD ${ }^{+} \mathrm{T}$ cells, macrophages and neutrophils, in STAD but no significant correlation with infiltrating level of $\mathrm{CD}^{+} \mathrm{T}$ cells and dendritic cells. 


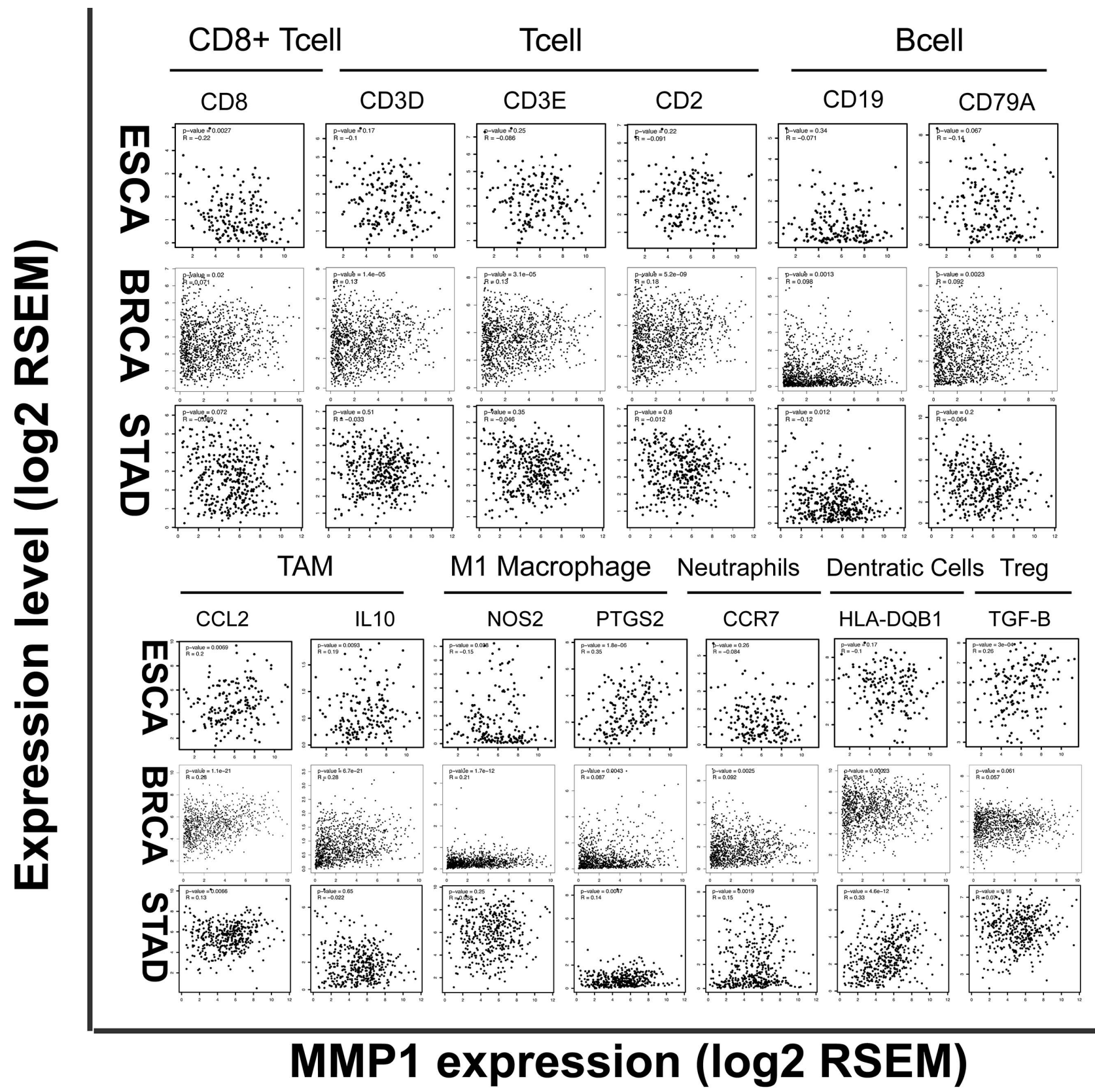

Figure 4. MMP1 expression correlated with macrophage polarization in BRCA, ESCA and STAD (stomach adenocarcinoma). Markers include CD8B of CD8 ${ }^{+} \mathrm{T}$ cell; CD3D, CD3E, CD2 of T cell; CD19, CD79A of B cell CCL2; and IL10 of TAMs (tumor-associated macrophages); NOS2, and PTGS2 of M1 macrophages; CCR7 of neutrophils; HLA-DQB1 of dendritic cell; TGF $\beta$ of Treg.

Table 2. Correlation analysis between MMP1 and relate genes and markers of immune cells in TIMER.

\begin{tabular}{|c|c|c|c|c|c|c|c|c|c|c|c|c|c|}
\hline \multirow{3}{*}{ Description } & \multirow{3}{*}{$\begin{array}{c}\text { Gene } \\
\text { markers }\end{array}$} & \multicolumn{4}{|c|}{ BRCA } & \multicolumn{4}{|c|}{ ESCA } & \multicolumn{4}{|c|}{ STAD } \\
\hline & & \multicolumn{2}{|c|}{ None } & \multicolumn{2}{|c|}{ Purity } & \multicolumn{2}{|c|}{ None } & \multicolumn{2}{|c|}{ Purity } & \multicolumn{2}{|c|}{ None } & \multicolumn{2}{|c|}{ Purity } \\
\hline & & Cor & $\mathrm{P}$ & Cor & $\mathrm{P}$ & Cor & $\mathrm{P}$ & Cor & $\mathrm{P}$ & Cor & $\mathrm{P}$ & Cor & $\mathrm{P}$ \\
\hline $\begin{array}{l}\mathrm{CD}^{+} \\
\mathrm{T} \text { cell }\end{array}$ & CD8B & 0.0670 & 0.0262 & 0.0629 & 0.0474 & -0.2090 & 0.0043 & -0.2523 & $* *$ & -0.1320 & 0.0071 & -0.1314 & 0.0104 \\
\hline
\end{tabular}


Continued

\begin{tabular}{|c|c|c|c|c|c|c|c|c|c|c|c|c|c|}
\hline & CD3D & 0.1411 & $* * *$ & 0.1433 & $* * *$ & -0.0822 & 0.2662 & -0.1553 & 0.0374 & -0.0169 & 0.7321 & -0.0458 & 0.3741 \\
\hline \multirow[t]{2}{*}{$\mathrm{T}$ cell } & CD3E & 0.1354 & $* * *$ & 0.1378 & $* * *$ & -0.0796 & 0.2813 & -0.1566 & 0.0358 & -0.0438 & 0.3734 & -0.0796 & 0.1221 \\
\hline & $\mathrm{CD} 2$ & 0.1823 & $* * *$ & 0.1911 & $* * *$ & -0.0954 & 0.1963 & -0.1620 & 0.0298 & -0.0143 & 0.7716 & -0.0430 & 0.4042 \\
\hline \multirow{2}{*}{ B cell } & CD19 & 0.1114 & ** & 0.1061 & ** & -0.1218 & 0.0986 & -0.1921 & 0.0098 & -0.1008 & 0.0402 & -0.1197 & 0.0198 \\
\hline & CD79A & 0.0993 & 0.0010 & 0.0929 & 0.0034 & -0.1296 & 0.0788 & -0.1996 & 0.0072 & -0.0449 & 0.3621 & -0.0775 & 0.1321 \\
\hline \multirow[b]{2}{*}{ Monocyte } & CD86 & 0.3261 & $* * *$ & 0.3388 & $* * *$ & 0.1881 & 0.0104 & 0.1348 & 0.0713 & 0.1766 & $* *$ & 0.1442 & 0.0049 \\
\hline & $\begin{array}{c}\text { CD115 } \\
\text { (CSF1R) }\end{array}$ & 0.1292 & $* * *$ & 0.1246 & $* *$ & 0.1329 & 0.0714 & 0.0887 & 0.2364 & 0.0796 & 0.1052 & 0.0483 & 0.3484 \\
\hline \multirow{3}{*}{ TAM } & CCL2 & 0.2955 & $* * *$ & 0.2941 & $* * *$ & 0.2009 & 0.0061 & 0.1664 & $* * *$ & 0.1194 & 0.0149 & 0.0892 & 0.0830 \\
\hline & CD68 & 0.3696 & $* * *$ & 0.3846 & $* * *$ & 0.1596 & 0.0300 & 0.1294 & $* * *$ & 0.1816 & ** & 0.1570 & 0.0022 \\
\hline & IL10 & 0.3334 & $* * *$ & 0.3457 & $* * *$ & 0.2242 & 0.0022 & 0.1972 & $* * *$ & 0.1405 & 0.0041 & 0.0939 & 0.0678 \\
\hline \multirow{3}{*}{ Marcophage } & $\begin{array}{l}\text { INOS } \\
\text { (NOS2) }\end{array}$ & 0.2002 & $* * *$ & 0.1912 & $* * *$ & -0.1455 & 0.0481 & -0.1446 & $* * *$ & 0.1398 & 0.0043 & 0.1396 & 0.0065 \\
\hline & IRF5 & 0.1114 & $* *$ & 0.1127 & $* *$ & -0.0193 & 0.7946 & -0.0515 & 0.4925 & -0.0669 & 0.1740 & -0.0760 & 0.1397 \\
\hline & PTGS2 & 0.0863 & 0.0042 & 0.0817 & 0.0100 & 0.3327 & $* * *$ & 0.3050 & $* * *$ & 0.3330 & $* * *$ & 0.3124 & $* * *$ \\
\hline \multirow{3}{*}{$\begin{array}{c}\text { M2 } \\
\text { Marcophage }\end{array}$} & CD163 & 0.3431 & $* * *$ & 0.3582 & $* * *$ & 0.1310 & 0.0755 & 0.0862 & 0.2498 & 0.1650 & $* *$ & 0.1295 & 0.0116 \\
\hline & VISG4 & 0.2502 & $* * *$ & 0.2605 & $* * *$ & 0.0926 & 0.2101 & 0.0507 & 0.4992 & 0.1019 & 0.0379 & 0.0684 & 0.1837 \\
\hline & MS4A4A & 0.2575 & $* * *$ & 0.2712 & $* * *$ & 0.0769 & 0.2981 & 0.0312 & 0.6773 & 0.0649 & 0.1871 & 0.0429 & 0.4048 \\
\hline Neutrophils & CCR7 & 0.0951 & 0.0016 & 0.0948 & 0.0028 & -0.0800 & 0.2789 & -0.1502 & 0.0441 & -0.0168 & 0.7335 & -0.0452 & 0.3798 \\
\hline \multirow{6}{*}{$\begin{array}{l}\text { Natural kill } \\
\text { cell }\end{array}$} & KIR2DL1 & 0.1538 & $* * *$ & 0.1546 & $* * *$ & -0.0530 & 0.4737 & -0.0858 & 0.2522 & 0.0187 & 0.7033 & 0.0512 & 0.3200 \\
\hline & KIR2DL3 & 0.1621 & $* * *$ & 0.1576 & *** & -0.0523 & 0.4793 & -0.0638 & 0.3949 & 0.0304 & 0.5374 & 0.0580 & 0.2598 \\
\hline & KIR2DL4 & 0.2156 & $* * *$ & 0.2160 & $* * *$ & -0.0688 & 0.3521 & -0.1022 & 0.1720 & 0.1601 & 0.0011 & 0.1434 & 0.0052 \\
\hline & KIR3DL2 & 0.1214 & $* * 1$ & 0.1255 & $* *$ & -0.0183 & 0.8045 & -0.0524 & 0.4844 & -0.0163 & 0.7400 & -0.0115 & 0.8238 \\
\hline & KIR3DL3 & 0.0890 & 0.0031 & 0.0917 & 0.0038 & -0.0435 & 0.5562 & -0.0549 & 0.4641 & 0.0582 & 0.2369 & 0.0847 & 0.0998 \\
\hline & KIR2DS4 & 0.1701 & $* * *$ & 0.1744 & $* * *$ & 0.0465 & 0.5294 & 0.0162 & 0.8296 & 0.0457 & 0.3529 & 0.0693 & 0.1782 \\
\hline \multirow{4}{*}{$\begin{array}{l}\text { Dendritic } \\
\text { cell }\end{array}$} & HLA-DPB1 & 0.0541 & 0.0729 & 0.0371 & 0.2423 & -0.0373 & 0.6143 & -0.0999 & 0.1819 & -0.0125 & 0.7995 & -0.0451 & 0.3815 \\
\hline & HLA-DQB1 & 0.1394 & $* * *$ & 0.1317 & $* * *$ & -0.1081 & 0.1429 & -0.1588 & 0.0332 & 0.0751 & 0.1266 & 0.0572 & 0.2668 \\
\hline & HLA-DRA & 0.1977 & $* * *$ & 0.2054 & $* * *$ & -0.0474 & 0.5220 & -0.1001 & 0.1811 & 0.0469 & 0.3406 & 0.0267 & 0.6049 \\
\hline & HLA-DPA1 & 0.1136 & $* *$ & 0.1071 & $* *$ & -0.0518 & 0.4836 & -0.1054 & 0.1592 & 0.0205 & 0.6767 & -0.0043 & 0.9341 \\
\hline \multirow{3}{*}{ Th1 } & STAT4 & 0.1423 & $* * *$ & 0.1485 & $* * *$ & 0.0918 & 0.2138 & 0.0214 & 0.7754 & -0.0223 & 0.6502 & -0.0431 & 0.4028 \\
\hline & STAT1 & 0.3206 & $* * *$ & 0.3236 & $* * *$ & 0.0495 & 0.5036 & 0.0130 & 0.8626 & 0.0773 & 0.1159 & 0.0723 & 0.1599 \\
\hline & IFN- $\gamma$ & 0.2338 & $* * *$ & 0.2368 & $* * *$ & 0.0282 & 0.7030 & -0.0218 & 0.7719 & 0.1110 & 0.0237 & 0.1006 & 0.0504 \\
\hline \multirow{3}{*}{ Th2 } & GATA3 & -0.3224 & *** & -0.3265 & $* * *$ & 0.0070 & 0.9250 & -0.0407 & 0.5879 & -0.1413 & 0.0039 & -0.1449 & 0.0047 \\
\hline & STAT6 & -0.2108 & $* * *$ & -0.2203 & $* * *$ & -0.0115 & 0.8761 & -0.0058 & 0.9380 & 0.0082 & 0.8675 & 0.0083 & 0.8716 \\
\hline & STAT5A & -0.1037 & ${ }^{* *} 6$ & -0.1201 & ** & 0.0488 & 0.5094 & 0.0167 & 0.8244 & 0.0370 & 0.4519 & 0.0397 & 0.4409 \\
\hline \multirow{2}{*}{ Tfh } & BCL6 & -0.0880 & 0.0035 & -0.0987 & 0.0018 & 0.0279 & 0.7064 & 0.0100 & 0.8943 & 0.0353 & 0.4735 & $-* * 2$ & 0.9974 \\
\hline & IL21 & 0.1499 & $* * *$ & 0.1506 & $* * *$ & -0.0226 & 0.7603 & -0.0743 & 0.3215 & 0.0307 & 0.5332 & 0.0137 & 0.7897 \\
\hline
\end{tabular}




\section{Continued}

\begin{tabular}{|c|c|c|c|c|c|c|c|c|c|c|c|c|c|}
\hline \multirow{2}{*}{ Th7 } & STAT3 & 0.0179 & 0.5523 & 0.0298 & 0.3472 & 0.0983 & 0.1831 & 0.0620 & 0.4083 & 0.0977 & 0.0467 & 0.0876 & 0.0887 \\
\hline & IL17A & 0.1467 & $* * *$ & 0.1566 & $* * *$ & 0.0768 & 0.2987 & 0.0816 & 0.2759 & 0.2263 & $* * *$ & 0.1965 & $* *$ \\
\hline \multirow{3}{*}{ Treg } & CCR8 & 0.3699 & $* * *$ & 0.3778 & $* * *$ & 0.1196 & 0.1050 & 0.0825 & 0.2710 & 0.1119 & 0.0226 & 0.1097 & 0.0328 \\
\hline & STAT5B & -0.2111 & $* * *$ & -0.2098 & $* * *$ & -0.0054 & 0.9901 & -0.0051 & 0.9457 & -0.0335 & 0.4964 & -0.0326 & 0.5270 \\
\hline & TGF $\beta$ & 0.5370 & $* * *$ & 0.5397 & $* * *$ & 0.4375 & $* * *$ & 0.4228 & $* * *$ & 0.1930 & $* *$ & 0.1731 & $* *$ \\
\hline \multirow{3}{*}{$\begin{array}{c}\text { T cell } \\
\text { exhaustion }\end{array}$} & CTLA4 & 0.2950 & $* * *$ & 0.3073 & $* * *$ & 0.0838 & 0.2570 & 0.0336 & 0.6543 & 0.0545 & 0.2676 & 0.0473 & 0.3581 \\
\hline & LAG3 & 0.2435 & $* * *$ & 0.2369 & $* * *$ & 0.0022 & 0.9762 & -0.0412 & 0.5831 & 0.0309 & 0.5298 & 0.0131 & 0.7988 \\
\hline & GZMB & 0.3106 & $* * *$ & 0.3282 & $* * *$ & 0.0107 & 0.8855 & -0.0431 & 0.5652 & 0.1848 & $* *$ & 0.1666 & 0.0011 \\
\hline
\end{tabular}

Table 3. Correlation analysis between MMP1 and relate genes and markers in GEPIA.

\begin{tabular}{|c|c|c|c|c|c|c|c|c|c|}
\hline \multirow{3}{*}{ Description } & \multirow{3}{*}{ Gene marker } & \multicolumn{4}{|c|}{ BRCA } & \multicolumn{4}{|c|}{ ESCA } \\
\hline & & \multicolumn{2}{|c|}{ Tumor } & \multicolumn{2}{|c|}{ Normal } & \multicolumn{2}{|c|}{ Tumor } & \multicolumn{2}{|c|}{ Normal } \\
\hline & & $\mathrm{R}$ & $\mathrm{P}$ & $\mathrm{R}$ & $\mathrm{P}$ & $\mathrm{R}$ & $\mathrm{P}$ & $\mathrm{R}$ & $\mathrm{P}$ \\
\hline $\mathrm{CD}^{+} \mathrm{T}$ cell & CD8B & 0.0570 & 0.0630 & 0.2300 & 0.0150 & -0.22 & 0.0027 & 0.13 & 0.66 \\
\hline \multirow{3}{*}{$\mathrm{T}$ cell } & CD3D & 0.1300 & 0.0000 & 0.3200 & 0.0005 & -0.0091 & 0.22 & 0.036 & 0.91 \\
\hline & CD3E & 0.1300 & 0.0000 & 0.2900 & 0.0017 & -0.0086 & 0.25 & 0.26 & 0.46 \\
\hline & $\mathrm{CD} 2$ & 0.1800 & 0.0000 & 0.2700 & 0.0039 & -0.1 & 0.17 & 0.35 & 0.24 \\
\hline \multirow{2}{*}{ B cell } & CD19 & 0.0980 & 0.0013 & 0.2000 & 0.0035 & -0.0071 & 0.34 & 0.071 & 0.82 \\
\hline & CD79A & 0.0920 & 0.0023 & 0.2600 & 0.0051 & -0.14 & 0.0067 & 0.066 & 0.83 \\
\hline \multirow{2}{*}{ TAM } & CCL2 & 0.2800 & 0.0000 & 0.0097 & 0.9200 & 0.2 & 0.0068 & -0.48 & 0.1 \\
\hline & IL10 & 0.2800 & 0.0000 & 0.1100 & 0.2500 & 0.19 & 0.0093 & -0.11 & 0.72 \\
\hline \multirow{2}{*}{ M1 Macrophage } & NOS2 & 0.2100 & 0.0000 & -0.0330 & 0.7300 & -0.15 & 0.0038 & 0.11 & 0.73 \\
\hline & PTGS2 & 0.0870 & 0.0043 & 0.0700 & 0.4700 & 0.35 & 0.0000 & -0.38 & 0.2 \\
\hline Neutrophils & CCR7 & 0.0920 & 0.0025 & 0.0000 & 0.4600 & -0.0084 & 0.26 & -0.14 & 0.68 \\
\hline Dendritic cells & HLA-DQB1 & 0.1100 & 0.0002 & 0.0330 & 0.2000 & -0.1 & 0.17 & -0.15 & 0.62 \\
\hline Treg & TGF $\beta$ & 0.0570 & 0.0600 & 0.0002 & 0.3500 & 0.26 & 0.000 & -0.52 & 0.074 \\
\hline
\end{tabular}

\section{Discussion}

Breast cancer is a heterogeneous and complex disease, which cause a huge health burden around the world, especially for women. Although remarkable improvements in early detection and personalized therapeutics have decreased mortality of BC in recent years, the prevention and treatment of breast cancer are still considerable public health concerns. Therefore, novel prognostic indicators are necessary to further improve the prognosis of breast cancer patients. MPs play an important role in the degradation of the stromal connective tissue and basement membrane components, which are key elements during tumor invasion and metastasis. Although the high expression of MMP1 was identified in several types of cancers, its expression status and prognostic merit in breast cancer still remain unclear. In addition, our research analysis the expression status of MMP1 
in breast cancer, then examines the importance of MMP1 in clinical and prognostic of breast cancer.

In this study, we examined the expression levels of MMP1 and its prognostic landscape in different types of cancers using independent datasets in Oncomine. Compared to normal tissues, MMP1 expression was higher in bladder, breast, cervical, colorectal, esophageal, gastric, head and neck, lung cancers, which consists of the published research. In addition, the TCGA data analysis showed that MMP1 expression was higher in BLCA, BRCA, CESC, CHOL, COAD, ESCA, GBM, HNSC, KIRC, LIHC, LUAD, LUSC, PAAD, READ, SKCM, STAD, THCA and UCEC, whereases expression was decreased in KICH and KIRP. The different expression levels in the two databases may be caused by data collection approach in different studies, but the higher MMP1 expression in most cancers is consistent in the two databases. Further study showed that MMP1 expression was related to a poor prognosis in breast, ovarian, head and neck, esophagus cancers by PrognoScan database. Meanwhile, the results were confirmed by KaplanMeier plotter database, that high MMP1 expression was significantly linked with a poorer prognosis in breast cancer, whereases the correlation between ovarian and gastric cancer were reduced. In addition, higher MMP1 expression was associated with worse OS and PFS in breast patients $(\mathrm{P}<0.05)$, and with ER positive, HER2 negative, intrinsic luminal A, lymph node status. Therefore, these results indicated that high LAYN expression is an independent risk factor and leads to a poor prognosis in breast patients.

Tumor-infiltrating immune cells are important in cancer patient prognosis and treatment efficacy [31] [32] [33]. The composition of immune cells in the tumor microenvironment contributes to tumor heterogeneity [34]. The results showed the MMP1 expression was significantly associated with the infiltration levels of $\mathrm{CD}^{+} \mathrm{T}$ cell, $\mathrm{CD} 8^{+} \mathrm{T}$ cell, macrophage, dendritic cell and NK cells in BRCA. In addition, the correlation between MMP1 expression and the marker genes of immune cells suggested that MMP1 can regulate tumor immunology in BRCA and ESCA. Specially, the strong correlation between CCL2, IL10 of TAMs, NOS2, PTGS2 of M1 macrophages and MMP1 expression indicated MMP1 may regulate the polarization of TAM. In addition, MMP1 can activate Tregs and induce $\mathrm{T}$ cell exhaustion, which is indicated by the correlation between MMP1 expression and FOXP3, CCR8, STAT5B and TGF $\beta$ of Treg and CTLA4, LAG3 and GZMB of T cell exhaustion. Meanwhile, the gene marker CD3D, CD3E, CD2 of $\mathrm{T}$ cell showed a strong correlation with MMP1 expression, but its correlation between CD19, CD79A of B cell were decreased.

In summary, high MMP1 expression significantly correlates with poor prognosis and increased immune infiltration levels in T Cell, B cell, macrophages, neutrophils and DCs of multiple cancers, especially in breast cancers. In addition, MMP1 expression potentially contributes to the regulation of tumor-associated macrophages (TAMs), DCs, T cell exhaustion, and Tregs. Therefore, LAYN likely plays an important role in immune cell infiltration and as a prognosis biomarker in patients with breast cancers. 


\section{Conflicts of Interest}

The authors declare no conflicts of interest regarding the publication of this paper.

\section{References}

[1] Bray, F., Ferlay, J., Soerjomataram, I., Siegel, R.L., Torre, L.A. and Jemal, A. (2018) Global Cancer Statistics 2018: GLOBOCAN Estimates of Incidence and Mortality Worldwide for 36 Cancers in 185 Countries. CA: A Cancer Journal for Clinicians, 68, 394-424. https://doi.org/10.3322/caac. 21492

[2] Alsop, B.R. and Sharma, P. (2016) Esophageal Cancer. Gastroenterology Clinics of North America, 45, 399-412. https://doi.org/10.1016/j.gtc.2016.04.001

[3] Kato, H. and Nakajima, M. (2013) Treatments for Esophageal Cancer: A Review. General Thoracic and Cardiovascular Surgery, 61, 330-335. https://doi.org/10.1007/s11748-013-0246-0

[4] Bollschweiler, E., Plum, P., Mönig, S.P., et al. (2017) Current and Future Treatment Options for Esophageal Cancer in the Elderly. Expert Opinion on Pharmacotherapy, 18, 1001-1010. https://doi.org/10.1080/14656566.2017.1334764

[5] Waks, A.G. and Winer, E.P. (2019) Breast Cancer Treatment: A Review. JAMA, 321, 288-300. https://doi.org/10.1001/jama.2018.19323

[6] Furrukh, M. and Qureshi, A. (2018) Treatment of Breast Cancer: Review and Updates. Journal of Ayub Medical College Abbottabad, 30, 264-274.

[7] Harbeck, N. and Gnant, M. (2017) Breast Cancer. The Lancet, 389, 1134-1150. https://doi.org/10.1016/S0140-6736(16)31891-8

[8] Thorat, M.A. and Balasubramanian, R. (2020) Breast Cancer Prevention in HighRisk Women. Best Practice \& Research Clinical Obstetrics \& Gynaecology, 65, 18-31. https://doi.org/10.1016/j.bpobgyn.2019.11.006

[9] Zhou, Z., Ma, X., Wang, F., Sun, L. and Zhang, G. (2018) A Matrix Metalloproteinase-1 Polymorphism, MMP1-1607 (1G>2G), Is Associated with Increased Cancer Risk: A Meta-Analysis Including 21,327 Patients. Disease Markers, 2018, Article ID: 7565834. https://doi.org/10.1155/2018/7565834

[10] Decock, J., Thirkettle, S., Wagstaff, L. and Edwards, D.R. (2011) Matrix Metalloproteinases: Protective Roles in Cancer. Journal of Cellular and Molecular Medicine, 15, 1254-1265. https://doi.org/10.1111/j.1582-4934.2011.01302.x

[11] Cui, N., Hu, M., and Khalil, R.A. (2017) Biochemical and Biological Attributes of Matrix Metalloproteinases. Progress in Molecular Biology and Translational Science, 147, 1-73. https://doi.org/10.1016/bs.pmbts.2017.02.005

[12] Murray, G.I., Duncan, M.E., O’Neil, P., McKay, J.A., Melvin, W.T. and Fothergill, J.E. (1998) Matrix Metalloproteinase-1 Is Associated with Poor Prognosis in Oesophageal Cancer. The Journal of Pathology, 185, 256-261. https://doi.org/10.1002/(SICI)1096-9896(199807)185:3\%3C256::AID-PATH115\%3E 3.0.CO;2-A

[13] Altadill, A., Rodríguez, M., González, L.O., Junquera, S., Corte, M.D., González-Dieguez, M.L., et al. (2009) Liver Expression of Matrix Metalloproteases and Their Inhibitors in Hepatocellular Carcinoma. Digestive and Liver Disease, 41, 740-748. https://doi.org/10.1016/j.dld.2009.01.016

[14] Inoue, T., Yashiro, M., Nishimura, S., Maeda, K., Sawada, T., Ogawa, Y., et al. (1999) Matrix Metalloproteinase-1 Expression Is a Prognostic Factor for Patients with Ad- 
vanced Gastric Cancer. International Journal of Molecular Medicine, 4, 73-80. https://doi.org/10.3892/ijmm.4.1.73

[15] Du, X., Wang, S., Lu, J., Cao, Y., Song, N., Yang, T., et al. (2011) Correlation between MMP1-PAR1 Axis and Clinical Outcome of Primary Gallbladder Carcinoma. Japanese Journal of Clinical Oncology, 41, 1086-1093.

https://doi.org/10.1093/jico/hyr108

[16] Mizrachi, A., Koren, R., Hadar, T., Yaniv, E., Morgenstern, S. and Shvero, J. (2011) Expression of MMP-1 in Invasive Well-Differentiated Thyroid Carcinoma. European Archives of Oto-Rhino-Laryngology, 268, 131-135. https://doi.org/10.1007/s00405-010-1343-7

[17] Ozden, F., Saygin, C., Uzunaslan, D., Onal, B., Durak, H. and Aki, H. (2013) Expression of MMP-1, MMP-9 and TIMP-2 in Prostate Carcinoma and Their Influence on Prognosis and Survival. Journal of Cancer Research and Clinical Oncology 139, 1373-1382. https://doi.org/10.1007/s00432-013-1453-x

[18] Murray, G.I., Duncan, M.E., O’Neil, P., Melvin, W.T. and Fothergill, J.E. (1996) Matrix Metalloproteinase-1 Is Associated with Poor Prognosis in Colorectal Cancer. Nature Medicine, 2, 461-462. https://doi.org/10.1038/nm0496-461

[19] Rhodes, D.R., Yu, J., Shanker, K., Deshpande, N., Varambally, R., Ghosh, D., et al. (2004) ONCOMINE: A Cancer Microarray Database and Integrated Data-Mining Platform. Neoplasia, 6, 1-6. https://doi.org/10.1016/S1476-5586(04)80047-2

[20] Rhodes, D.R., Kalyana-Sundaram, S., Mahavisno, V., Varambally, R., Yu, J., Briggs, B.B., et al. (2007) Oncomine 3.0: Genes, Pathways, and Networks in a Collection of 18,000 Cancer Gene Expression Profiles. Neoplasia, 9, 166-180.

https://doi.org/10.1593/neo.07112

[21] Mizuno, H., Kitada, K., Nakai, K. and Sarai, A. (2009) PrognoScan: A New Database for Meta-Analysis of the Prognostic Value of Genes. BMC Medical Genomics, 2, Article No. 18. https://doi.org/10.1186/1755-8794-2-18

[22] Yuan, Q., Sun, N., Zheng, J., Wang, Y., Yan, X., Mai, W., Liao, Y. and Chen, X. (2020) Prognostic and Immunological Role of FUN14 Domain Containing 1 in Pan-Cancer: Friend or Foe? Frontiers in Oncology, 10, Article No. 1502.

https://doi.org/10.3389/fonc.2019.01502

[23] Györffy, B., Lanczky, A., Eklund, A.C., Denkert, C., Budczies, J., Li, Q., et al. (2010) An Online Survival Analysis Tool to Rapidly Assess the Effect of 22,277 Genes on Breast Cancer Prognosis Using Microarray Data of 1,809 Patients. Breast Cancer Research and Treatment, 123, 725-731. https://doi.org/10.1007/s10549-009-0674-9

[24] Győrffy, B., Surowiak, P., Budczies, J. and Lánczky, A. (2013) Online Survival Analysis Software to Assess the Prognostic Value of Biomarkers Using Transcriptomic Data in Non-Small-Cell Lung Cancer. PLoS ONE, 8, Article ID: e82241.

https://doi.org/10.1371/journal.pone.0082241

[25] Gyorffy, B., Lánczky, A. and Szállási Z. (2012) Implementing an Online Tool for Genome-Wide Validation of Survival-Associated Biomarkers in Ovarian-Cancer Using Microarray Data from 1287 Patients. International Journal of Molecular Medicine, 19, 197-208. https://doi.org/10.1530/ERC-11-0329

[26] Szász, A.M., Lánczky, A., Nagy, Á., Förster, S., Hark, K., Green, J.E., et al. (2016) Cross-Validation of Survival Associated Biomarkers in Gastric Cancer Using Transcriptomic Data of 1,065 Patients. Oncotarget, 7, 49322-49333.

https://doi.org/10.18632/oncotarget.10337

[27] Li, T., Fu, J., Zeng, Z., Cohen, D., Li, J., Chen, Q., et al. (2020) TIMER2.0 for Analysis of Tumor-Infiltrating Immune Cells. Nucleic Acids Research, 48, W509-W514. 
https://doi.org/10.1093/nar/gkaa407

[28] Tang, Z., Li, C., Kang, B., Gao, G., Li, C. and Zhang, Z. (2017) GEPIA: A Web Server for Cancer and Normal Gene Expression Profiling and Interactive Analyses. Nucleic Acids Research, 45, W98-W102. https://doi.org/10.1093/nar/gkx247

[29] Tsang, J.Y.S. and Tse, G.M. (2020) Molecular Classification of Breast Cancer. Advances in Anatomic Pathology, 27, 27-35. https://doi.org/10.1097/PAP.0000000000000232

[30] Mook, S., Schmidt, M.K., Rutgers, E.J., van de Velde, A.O., Visser, O., Rutgers, S.M., et al. (2009) Calibration and Discriminatory Accuracy of Prognosis Calculation for Breast Cancer with the Online Adjuvant! Program: A Hospital-Based Retrospective Cohort Study. The Lancet Oncology, 10, 1070-1076.

https://doi.org/10.1016/S1470-2045(09)70254-2

[31] Denkert, C., von Minckwitz, G., Darb-Esfahani, S., Lederer, B., Heppner, B.I., Weber, K.E., et al. (2018) Tumour-Infiltrating Lymphocytes and Prognosis in Different Subtypes of Breast Cancer: A Pooled Analysis of 3771 Patients Treated with Neoadjuvant Therapy. The Lancet Oncology, 19, 40-50. https://doi.org/10.1016/S1470-2045(17)30904-X

[32] Denkert, C., Loibl, S., Noske, A., Roller, M., Müller, B.M., Komor, M., et al. (2010) Tumor-Associated Lymphocytes as an Independent Predictor of Response to Neoadjuvant Chemotherapy in Breast Cancer. Journal of Clinical Oncology, 28, 105-113. https://doi.org/10.1200/JCO.2009.23.7370

[33] Savas, P., Salgado, R., Denkert, C., Sotiriou, C., Darcy, P.K., Smyth, M.J., et al. (2016) Clinical Relevance of Host Immunity in Breast Cancer: From TILs to the Clinic. Nature Reviews Clinical Oncology, 13, 228-241.

https://doi.org/10.1038/nrclinonc.2015.215

[34] McGranahan, N. and Swanton, C. (2017) Clonal Heterogeneity and Tumor Evolution: Past, Present, and the Future. Cell, 168, 613-628.

https://doi.org/10.1016/j.cell.2017.01.018 